\title{
The Adhesion and Diffusion of Saturate, Asphaltene, Resin and Aromatic (SARA) Molecules on Oxygenated and Hydrogenated Carbon Nanotubes (CNTs)
}

\author{
Mehdi Shishehbor ${ }^{1}$, Hadi S. Esmaeeli ${ }^{2, *}$ and M. Reza Pouranian ${ }^{3}$ \\ 1 Department of Civil and Environmental Engineering, Henry Samueli School of Engineering, \\ University of California Irvine, E4130 Engineering Gateway, Irvine, CA 92697, USA; mshisheh@uci.edu \\ 2 Department of Civil and Environmental Engineering, Princeton University, Princeton, NJ 08544, USA \\ 3 School of Civil Engineering, Purdue University, West Lafayette, IN 47907, USA; mpourani@purdue.edu \\ * Correspondence: hs6710@princeton.edu
}

check for

updates

Citation: Shishehbor, M.; Esmaeeli, H.S.; Pouranian, M.R. The Adhesion and Diffusion of Saturate, Asphaltene, Resin and Aromatic (SARA)

Molecules on Oxygenated and Hydrogenated Carbon Nanotubes (CNTs). Infrastructures 2021, 6, 123. https://doi.org/10.3390/

infrastructures6090123

Academic Editor: Md. Safiuddin

Received: 30 July 2021

Accepted: 29 August 2021

Published: 1 September 2021

Publisher's Note: MDPI stays neutral with regard to jurisdictional claims in published maps and institutional affiliations.

Copyright: (c) 2021 by the authors. Licensee MDPI, Basel, Switzerland. This article is an open access article distributed under the terms and conditions of the Creative Commons Attribution (CC BY) license (https:/ / creativecommons.org/licenses/by/ $4.0 /)$.

\begin{abstract}
The interfacial adhesion between asphalt binder and carbon nanotubes (CNTs) depends on many nanoscopic properties such as diffusion of SARA molecules on CNTs surface. Functionalization of CNTs with Oxygens ( $\mathrm{O}=\mathrm{CNTs})$, hydroxyl groups (HO-CNTs), and hydrogens (H-CNTs) has been an effective way to modify the surface properties of CNTs and ultimately the macroscopic properties of the CNT-composites. This paper presents the effect of different dosages of oxygenated and hydrogenated CNTs on the adhesion and diffusion of SARA molecules on CNTs' surfaces. First, reactive molecular dynamics simulation is used to oxygenate and hydrogenate CNTs up to a certain dosage. Next, it is employed to model the interaction and diffusion of SARA molecules with the functionalized CNTs. We employ the steer molecular dynamic (SMD) and Einstein formula to calculate the adhesion and diffusion properties. The results demonstrate that hydrogenation has little effect on the adhesion energy, while oxygenation can increase adhesion energy up to $100 \%$ for $25 \%$ dosage. The diffusion coefficient dramatically drops for both oxygenated and hydrogenated CNTs, with lower values for the latter. We observe that for hydrogenated and oxygenated CNTs at different dosages, asphaltene, resin, aromatic, and saturate molecules have the highest to lowest values, respectively.
\end{abstract}

Keywords: diffusion; molecular dynamics; SARA molecules; adhesion; carbon nanotube

\section{Introduction}

Since their discovery in 1991, many researchers have extensively studied carbon nanotubes (CNTs) for their exceptional properties such as low density, high mechanical properties, and outstanding electronic properties [1]. CNTs can be imagined to be formed by rolling up a graphene sheet (a two-dimensional hexagonal lattice of carbon atoms in one layer) into a cylindrical shell. Each carbon atom in CNTs is connected to three neighboring atoms ( $\cong 0.146 \mathrm{~nm} \mathrm{C}-\mathrm{C}$ bond length), forming sp ${ }^{2}$ hybridized atomic structures. Studies have shown that the properties of CNTs can be tailored with surface functionalization [2] for different applications such as drug delivery and biosensors [3]. Functionalization of CNTs with oxygens $(\mathrm{O}=\mathrm{CNTs})$, hydroxyl groups $(\mathrm{HO}-\mathrm{CNTs})$, and hydrogens $(\mathrm{H}-\mathrm{CNTs})$ have been introduced by many researchers for different applications such as purification, biosensor [4-6], hydrogen storage, and semiconductors [7-10]. The functionalized atoms such as oxygens change the intrinsic properties of CNTs through change in sp2 atomic structure and improve interfacial adhesion and solubility [2]. In recent years, there has been a great interest in employing CNT-, graphene-, graphene oxide (GO)- and polymer grafted graphene as modifiers to improve fatigue life and performance of asphalt binders [11-15]. A study on the intrinsic healing capacity of GO-modified asphalt binder showed that increasing the dosage of GO improves thermodynamic properties and self-healing capacity 
of aged binder [15]. While exceptional properties of CNTs can improve the performance of CNT-modified asphalt binders, weak interfacial properties and load transfer at the interface can dramatically decrease their properties [16,17]. Functionalized CNTs, however, can enhance interfacial properties and bring CNTs closer to their full potential. Most studies on the functionalized surface of $\mathrm{CNT}$ can be related to the usage of graphene oxide (GO) $[15,18]$, which has similar chemistry in asphalt binder. However, there have been few studies on the usage of functionalized CNT (with 3\% carboxylic acid groups) in asphalt binder [19]. Previous studies have shown that the contact and diffusion of molecules at the interface are important factors behind the molecular adhesion of materials [20-24]. Therefore, the aggregation and distribution of saturate, asphaltene, resin, and aromatic (SARA) molecules on the surface of inclusions (CNTs, aggregates, etc.) are crucial in defining the adhesion behaviors of the asphalt-inclusion interface. For example, Huang et al. studied the diffusion of SARA molecules on mineral surfaces. They showed that the adhesion between asphalt and aggregate could be improved via the diffusion of SARA molecules on the aggregate surface [20]. A study by Luo et al. on the diffusion of SARA molecules on the mineral aggregate demonstrated that the diffusion of SARA molecules on the $\mathrm{Al}_{2} \mathrm{O}_{3}$ aggregate could be independent of temperature due to the interaction between asphalt components [25]. In recent years, many researchers have employed molecular dynamic (MD) to study the properties of the binder at the nanoscale $[17,26]$, diffusion of SARA molecules on various aggregates [20,23-25,27-29], interactions of GrapheneSARA [30], the interactions of SARA-CNTs and CNTs-aggregate [16,31].

In this study, the effect of oxygenation and hydrogenation of CNTs on adhesion and diffusion of saturate, asphaltene, resin, and aromatic (SARA) molecules on CNTs surface is evaluated. The results are compared with those in pristine CNTs using reactive molecular dynamic (MD) simulations. MD simulations provide a new perspective and understanding at the nanoscale that can hardly be achieved through experiments. A combination study of diffusion and adhesion of different SARA molecules provides more insight into the cause of adhesion (kinetic vs. static) between CNTs and SARA molecules. This analysis is particularly important as the adhesion provides information about the separation of surfaces (normal direction) while diffusion mostly provides friction strength. The result of this work helps to design a better material according to the adhesion and diffusion response of different molecules. From the cost perspective, both CNT and graphene sheets are expensive materials. Studies have suggested limiting the usage of pristine CNT to $1.5 \%$ to justify the cost [32]. However, one of the advantages of GO and functionalized CNT is reducing the cost. Functionated CNT and GO can be easily separated in the solution due to their hydrophilic attributes and therefore reduce their cost [33].

\section{Molecular Models for SARA-CNT Interface}

The SARA molecules used in this study (shown in Figure 1a-d) are suggested by Li and Greenfield [17,34] and used in a previous study for SARA CNTs interactions [16,17]. The SARA molecules have many potential choices, and the selected molecules do not represent all possible choices. However, we believe each molecule represents its category (saturate, asphaltene, resin, and aromatic molecules). To make a SARA-CNT interface, each SARA molecule is placed within $5 \AA$ distance from the surface of a CNT $(10,10)$ (with $100 \AA$ length as shown in Figure 2 for aromatic molecule). Next, a vacuum layer of $50 \AA$ is added in the $\mathrm{x}$-direction to prevent the interaction of the molecules with periodic images in the pulling direction. Although in many cases, especially in the crude oil industry, the SARA molecules are dispersed in fluids, in nanocomposite studies (for instance, the interaction between asphalt binder and carbon nanotube), the SARA molecules are in direct contact with other materials such as carbon nanotube. Therefore the vacuum environment is more appropriate. 


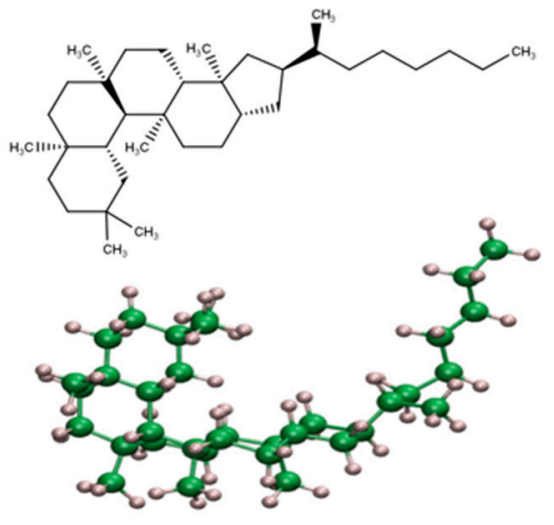

(a) Molecular model of Saturate

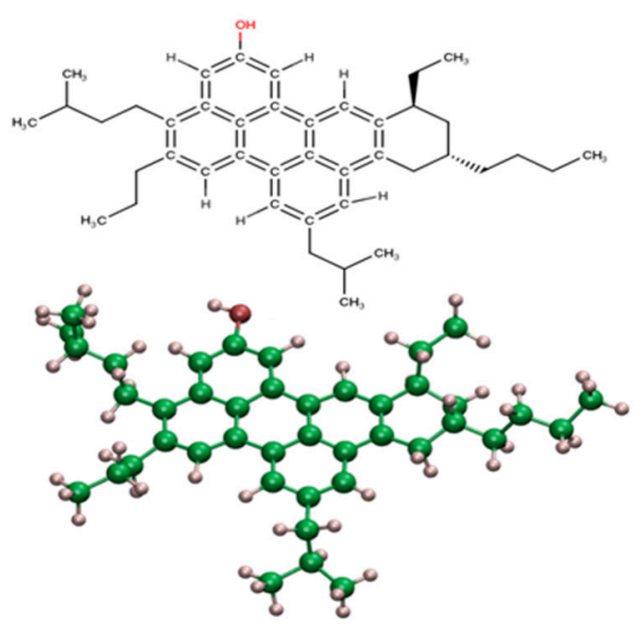

(b) Molecular model of Asphaltene<smiles>CCCCCc1ccc2nc3c(c(C)c2c1)[C@H]1CC[C@@]2(C)[C@H](CC[C@@]4(C)[C@@H]2CC[C@]2(C)CC(C)(C)CC[C@H]24)[C@@]1(C)C3</smiles>

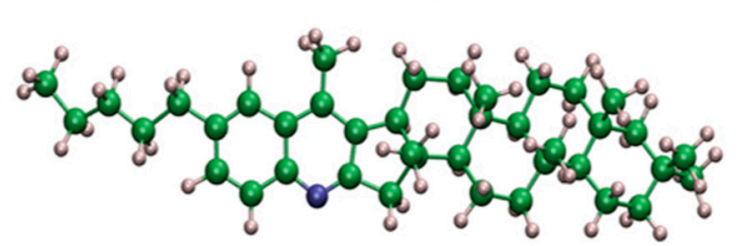

(c) Molecular model of Resin

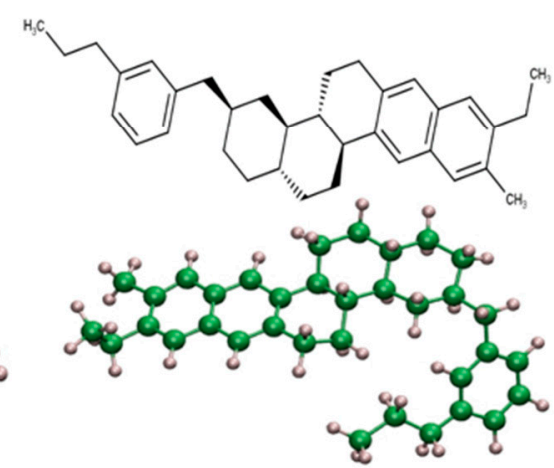

(d) Molecular model of Aromatic

Figure 1. Molecular structure of SARA molecules: (a) saturate, (b) asphaltene, (c) resin and (d) aromatic (adapted from [27]).

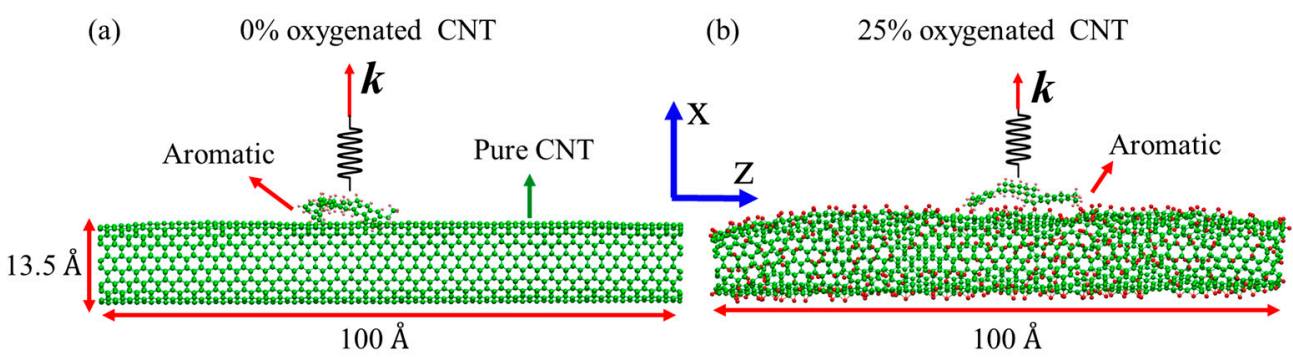

Figure 2. Molecular dynamic set up of Aromatic molecule on the surface of CNT before steering and after equilibration. (a) $0 \%$ oxygenated $\mathrm{CNT}$ and (b) $25 \%$ oxygenated CNT.

\subsection{Force Field}

Generally, force fields can be categorized into nonreactive and reactive force fields. Although the nonreactive force fields are computationally fast compared to reactive force fields, they cannot model bond breaking and new bond formation. On the other hand, in reactive force fields such as REAXFF, the bonds are defined based on the bond order concepts, and bond breaking and formation are possible. Furthermore, as opposed to nonreactive force fields, the charge of atoms is not constant, and it can be updated at each time step. Finally, REAXFF provides explicit hydrogen bonding (HB) potential term and the possibility of studying the effect of $\mathrm{HB}$ on the interface properties, while in most nonreactive force fields, HB is included in the electrostatic (ES) interactions. 


\subsection{Molecular Model for Hydrogenated and Oxygenated CNTs}

To generate hydrogenated and oxygenated CNTs, different dosages (0-25\%) of hydrogen and oxygen are randomly placed near carbon atoms in CNT and equilibrated for 100 ps in NVT ensemble at room temperature using Nosé-Hoover thermostat. Note that $100 \mathrm{ps}$ is the time required for bond formation between deposited oxygens/hydrogens with CNT. Further equilibration is performed later for the CNT-SARA molecules system, as explained in Section 3. Due to the dynamic bond formation/breaking nature of REAXFF, any hydrogens or oxygens that are not energetically stable in the initial configuration will be automatically removed. LAMMPS package [35] and ReaxFF Forcefield [36] were employed to perform all the simulations. All the atomistic images were prepared with VMD atomistic visualizations package [37].

\section{Simulation and Analysis Protocol for SARA-CNT System}

3.1. Molecular Dynamics (MD) Protocol

After constructing the model of hydrogenated and oxygenated SARA-CNT, the system is minimized by using a conjugate gradient (CG) and Hessian-free truncated Newton algorithm ( $\mathrm{hftn}$ ) methods for 100,000 steps and then is equilibrated at $300 \mathrm{~K}$ (room temperature) for 1000 ps with 0.25 fs timestep in NVT ensemble using Nosé-Hoover thermostat. Note that the purpose of the minimization step is merely avoiding instability at the beginning of NVT rather than reaching the minimum energy; therefore, any minimization method such as steepest descent should also work. LAMMPS package [35] and ReaxFF Forcefield [36] were employed to perform all the simulations. All the atomistic images were prepared with VMD atomistic visualizations package [37].

\subsection{Diffusion Analysis}

Here, we use the diffusion coefficient to characterize the diffusivity of SARA molecules on hydrogenated and oxygenated CNT. In MD simulations, the diffusion coefficient $(D)$ can be obtained from the slope of the mean square displacement (MSD) time curves through the Einstein formula [20].

$$
D=\frac{1}{2 n} \lim _{t \rightarrow \infty} \frac{1}{t} M S D(t)
$$

where $D$ is diffusion coefficient, $\mathrm{n}$ is the dimension ( $n=3$ here), $t$ is time, and $M S D(t)$ is mean square displacement defined as follow:

$$
M S D=\frac{1}{N} \sum_{n=1}^{N}\left(x_{n}(t)-x_{n}(0)\right)^{2}
$$

where $N$ is the number of particles and $x$ is the position of particles. To obtain MSD-t curves for each case, $100 \mathrm{ps}$ MD simulation was performed after the initial 1000 ps equilibration, and then the slope of MSD-t curves was obtained by simple linear regression. Here, although the center of mass of SARA molecules moves on a surface (CNT), the surface is $3 \mathrm{D}$, and the center of mass moves in a 3D space and therefore, we have chosen $n=3$ in Equation (2). We have to mention that the choice of $n=2$ or $n=3$ will not significantly change the diffusion coefficient values, and here, the relative comparison between the case studies is more important. In the case studies where the molecules move inside the CNT (confined system), the interaction of molecules with the wall becomes important, and Equation (2) should be adjusted accordingly.

\subsection{Adhesion Analysis}

Steer molecular dynamic (SMD) simulations are used to obtain the potential of mean force (PMF) for hydrogenated and oxygenated SARA-CNT. In the SMD approach, a driving force pulls a group of atoms toward a chosen coordinates and provides nonequilibrium trajectories along the coordinate. Then, in the SMD method, PMF (adhesion energy) is obtained through averaging the integral of steering force in the direction of the pulling ( $x$ - 
direction) over many different trajectories paths (five paths in this work) [38]. The value of spring constant and velocity for all SMD case studies of this work are $k=100 \mathrm{kcal} / \mathrm{mol} \AA^{2}$ and $5 \mathrm{~m} / \mathrm{s}$, respectively (Figure 2). Figure 2 demonstrates the steering of Aromatic molecules away from the surface of CNT with $0 \%$ (Figure 2a) and 25\% (Figure 2b) oxygenation, respectively.

In SMD simulations, the adhesion energy $\left(E_{a}\right)$ can be calculated by averaging the integral of pulling force $\left(F_{x}\right)$ in the direction of the pulling, $x$, over many different trajectories (5 samples in this work) and using second-order cumulant expansion, which approximates Jarzynski's equality [38] as shown in Equations (3) and (4). Another approach can also obtain the adhesion energy by subtracting the total energy of a complex system (CNTSARA) from their isolated energy (CNT and SARA molecules) [29]. The work of adhesion $(W)$ and the strength can then be obtained by dividing the adhesion energy and the maximum force of pulling over the effective area $(A)$ of SARA components (Table 1), respectively.

$$
\begin{gathered}
\sum=\int F_{x} \cdot d x \\
E_{a}=<\sum>-\frac{\beta}{2}\left(<\sum^{2}>-<\sum>^{2}\right), \beta=\frac{1}{k_{b} T} \\
W=\frac{E_{a}}{A} \\
\sigma=\frac{F_{m}}{A}
\end{gathered}
$$

where $\sum$ is the cumulative work, $\beta=1 / k_{b} T$, $\sigma$ is the strength, $F_{m}$ is the maximum pulling force.

Table 1. The number of atoms, molecular weight, and surface area of SARA components (adapted from [27]).

\begin{tabular}{cccc}
\hline Molecule & Number of Atoms & Molecular Weight (amu) & Molecular Area $\left(\AA^{\mathbf{2}}\right)$ \\
\hline Saturate & 97 & 480 & 170 \\
Asphaltene & 97 & 575 & 255 \\
Resin & 100 & 555 & 200 \\
Aromatic & 79 & 465 & 205 \\
\hline
\end{tabular}

\section{Results}

This section demonstrates the results for generated hydrogenated and oxygenated CNTs (procedure explained in Section 2). Next, the results obtained from the simulation of adhesion and diffusion of SARA molecules on the surface of oxygenated and hydrogenated CNT are compared (the procedure is explained in Section 3).

Figure 3 a displays the variation of the MSD with respect to time over the $1000 \mathrm{ps}$ time span for an aromatic molecule on CNT with $0 \%$ functionalization. The MSD curve shows the equilibrium in the system after 500 ps as the values fluctuate around $70 \AA^{2}$. The temperature and potential energy variation during 1000 ps simulation (shown in Figure $3 b$ with red and green lines, respectively) show fluctuation around the equilibrium value, indicating equilibrium state. 


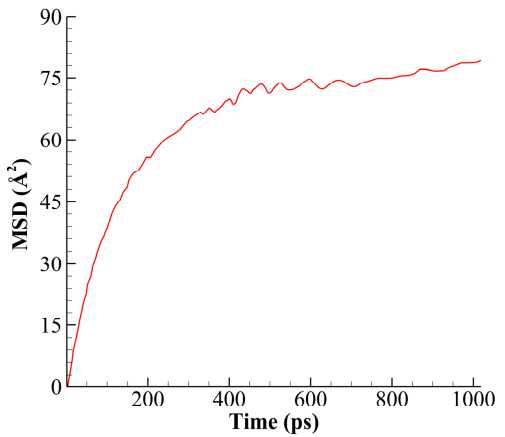

(a)

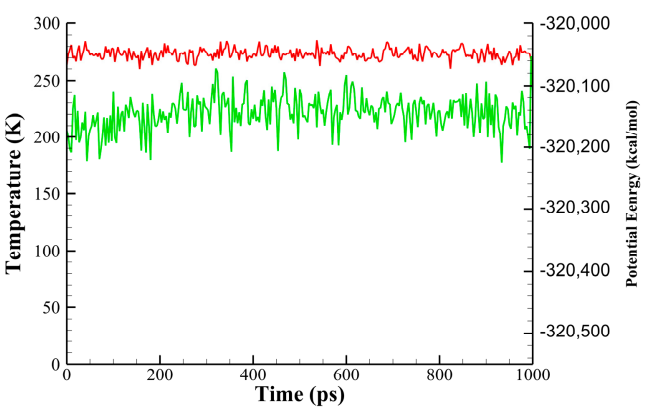

(b)

Figure 3. The change of (a) mean square displacement (MSD) and (b) temperature and potential energy during $1000 \mathrm{ps}$ is shown.

The generated dosage of 3-25\% hydrogenated and oxygenated CNTs are shown in Figures $4 a-d$ and $5 b-d$, respectively. Our simulation shows that above $25 \%$ dosage of randomly positioned hydrogens and oxygens, CNTs becomes unstable, CNTs do not remain as a tube anymore, and several $\mathrm{C}-\mathrm{C}$ bonds break. Therefore our results are limited to a maximum $25 \%$ dosage. We use similar oxygenated and hydrogenated CNTs structures for each SARA molecule to avoid random oxygenated and hydrogenated effects on the results. As mentioned in Section 3, after constructing the hydrogenated and oxygenated CNTs, each SARA molecule is placed on the CNT surface, and a 1000 ps MD simulation is performed. The final structure after $1000 \mathrm{ps}$ MD simulations of an aromatic molecule on $3-25 \%$ hydrogenated and oxygenated CNTs are shown in Figures $4 a-d$ and $5 b-d$, respectively.

(a) $3 \%$

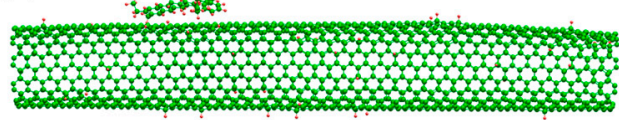

(c) $12 \%$

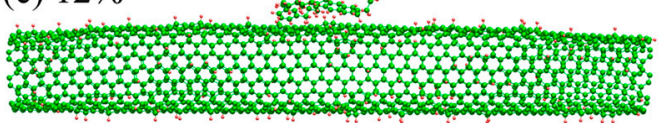

(b) $6 \%$

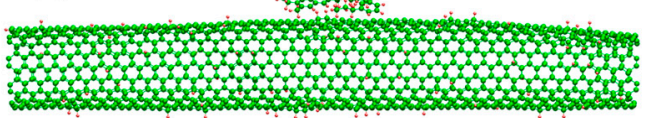

(d) $25 \%$

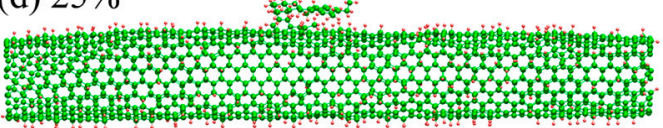

Figure 4. Molecular structure of aromatic molecule and CNTs with different hydrogenated dosage of (a) $3 \%$, (b) $6 \%$, (c) $12 \%$, and (d) $25 \%$.

(a) $3 \%$

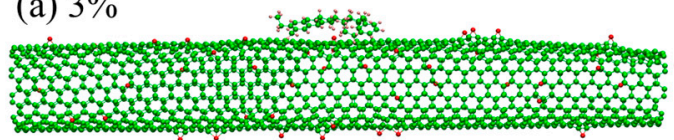

(c) $12 \%$

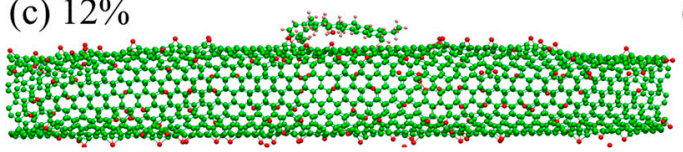

(b) $6 \%$

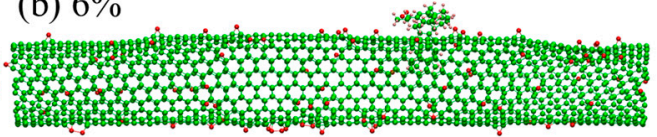

(d) $25 \%$

Figure 5. Molecular structure of aromatic molecule and CNTs with different oxygenated dosage of (a) $3 \%$, (b) $6 \%$, (c) $12 \%$, and (d) $25 \%$. 
Figure 6 compares the adhesion of SARA molecules for different dosages of hydrogenated and oxygenated CNTs. The adhesion results for hydrogenated CNTs do not show significant variation with changing hydrogen dosage on CNTs (Figure 6a). Among SARA molecules, the asphaltene molecule has the highest adhesion with $55 \pm 5 \mathrm{kcal} / \mathrm{mol}$, resin molecule with $45 \pm 5 \mathrm{kcal} / \mathrm{mol}$, and then aromatic molecule with $40 \pm 3 \mathrm{kcal} / \mathrm{mol}$, and finally the saturate molecule has the lowest adhesion with $30 \pm 5 \mathrm{kcal} / \mathrm{mol}$ as shown in Figure 6a. For oxygenated CNTs, the result demonstrates that the adhesion of SARA molecules increases significantly by increasing the oxygenation dosage, as shown in Figure $6 \mathrm{~b}$. For the asphaltene molecule, the blue region in Figure $6 \mathrm{~b}$, adhesion increases from $50 \pm 5 \mathrm{kcal} / \mathrm{mol}$ for $0 \%$ dosage to $75 \pm 5 \mathrm{kcal} / \mathrm{mol}$ at $12 \%$ oxygenation and remain constant at $75 \pm 5 \mathrm{kcal} / \mathrm{mol}$ for higher dosage $(25 \%)$. Adhesion of the resin molecule on the surface of an oxygenated CNTs is improved by $50 \%$ for $25 \%$ dosage as the values change from $45 \pm 2 \mathrm{kcal} / \mathrm{mol}$ at $0 \%$ dosage to $70 \pm 2 \mathrm{kcal} / \mathrm{mol}$ at $25 \%$ dosage (shown with the green region in Figure 6b). Aromatic molecule adhesion values, shown with the red region in Figure $6 \mathrm{~b}$, show a similar trend observed in resin molecule and is increased from $40 \pm 2 \mathrm{kcal} / \mathrm{mol}$ at $0 \%$ dosage to $75 \pm 2 \mathrm{kcal} / \mathrm{mol}$ at $25 \%$ dosage ( $45 \%$ increase). However, the best enhancement is observed in saturate molecule, shown with the black region in Figure $6 \mathrm{~b}$, with a $100 \%$ increase in adhesion at $25 \%$ dosage $(60 \pm 4 \mathrm{kcal} / \mathrm{mol})$. To demonstrate more clearly how hydrogenated and oxygenated change the adhesion properties for SARA molecules, both results have been shown together in Figure $6 \mathrm{c}$ with a dashed line representing hydrogenated CNTs and a solid line representing oxygenated CNTs.

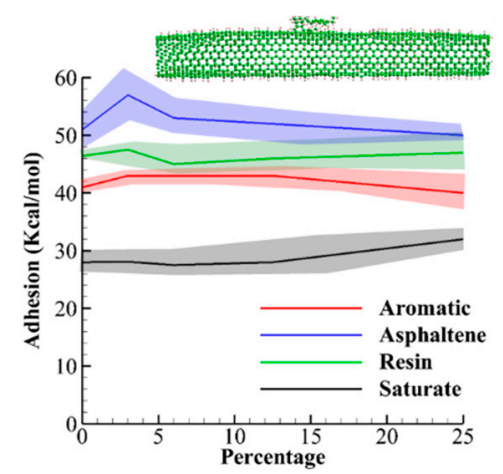

(a)

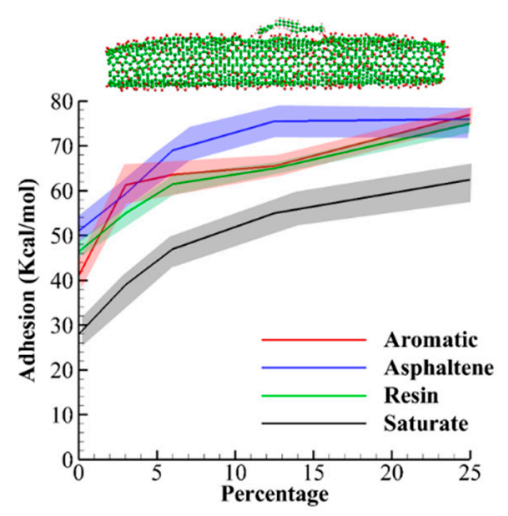

(b)

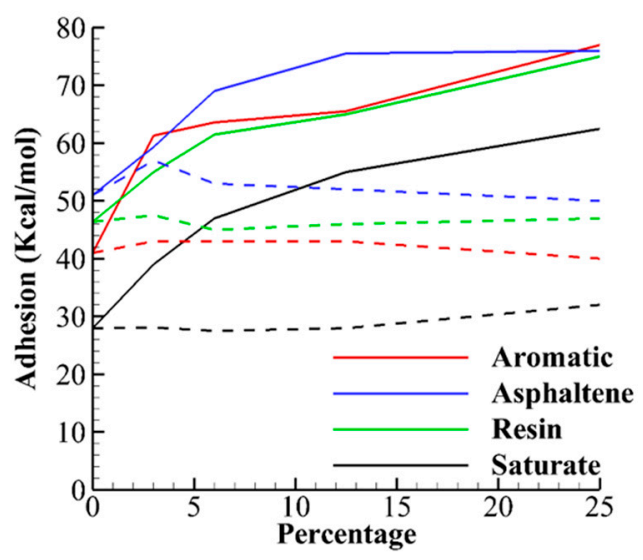

(c)

Figure 6. Adhesion energy of SARA and different dosages of hydrogenated and oxygenated CNTs. (a) SARA-hydrogenated CNTs, (b) SARA-oxygenated CNTs, and (c) comparison of SARAhydrogenated (dashed lines) and SARA-oxygenated CNTs (solid lines). 
The diffusion of SARA molecules on the surface of CNTs with different dosages of hydrogenation and oxygenation is shown in Figure 7. The diffusion of SARA on hydrogenated CNTs shows a sudden drop in diffusion coefficient by increasing hydrogenation dosage (Figure 7a). The diffusion coefficient of saturate molecule reduces from $2 \pm 0.2 \times 10^{-10} \mathrm{~m}^{2} / \mathrm{s}$ at $0 \%$ hydrogen dosage to $0.05 \pm 0.05 \times 10^{-10} \mathrm{~m}^{2} / \mathrm{s}$ at $25 \%$ dosage. As expected, the values for diffusion coefficient for saturate molecule is higher than the other components as smaller interatomic forces (adhesion) lead to more movement of molecules on the surface of CNTs. For asphaltene molecules, although the diffusion coefficient is higher at $0 \%\left(1.6 \pm 0.1 \times 10^{-10} \mathrm{~m}^{2} / \mathrm{s}\right)$ than resin and aromatic molecules, the values drop more rapidly. After $6 \%$ dosage, they are below those in resin and aromatic molecules. After saturate, and asphaltene molecules, aromatic molecule (red region in Figure $7 \mathrm{a})$ has the highest initial diffusion coefficient at $0 \%\left(1.4 \pm 0.1 \times 10^{-10} \mathrm{~m}^{2} / \mathrm{s}\right)$, but after a sudden drop to $0.4 \pm 0.1 \times 10^{-10} \mathrm{~m}^{2} / \mathrm{s}$ at $3 \%$ dosage, the values are smoothly reduced to $0.25 \pm 0.1 \times 10^{-10} \mathrm{~m}^{2} / \mathrm{s}$ at $25 \%$ dosage. Finally, although resin molecule has the lowest initial diffusion coefficient at $0 \%$ dosage $\left(1.1 \pm 0.1 \times 10^{-10} \mathrm{~m}^{2} / \mathrm{s}\right)$, it has the smoothest drop in diffusion coefficient, and they values merge to $0.25 \pm 0.1 \times 10^{-10} \mathrm{~m}^{2} / \mathrm{s}$ at $25 \%$ dosage (green region). The diffusion of SARA on oxygenated CNTs is shown in Figure 7b. The results show a more sudden drop in diffusion coefficient than observed in hydrogenated CNTs. This alteration is due to higher interatomic forces between SARA and oxygenated CNTs. The diffusion coefficient of saturate molecule reduces from $2 \pm 0.2 \times 10^{-10} \mathrm{~m}^{2} / \mathrm{s}$ at $0 \%$ hydrogen dosage to $0.4 \pm 0.1 \times 10^{-10} \mathrm{~m}^{2} / \mathrm{s}$ at $3 \%$ dosage and then smoothly reduces to $0.001 \pm 0.001 \times 10^{-10} \mathrm{~m}^{2} / \mathrm{s}$ at $25 \%$ dosage. The diffusion coefficient values for asphaltene, resin, and aromatic molecules on oxygenated CNTs with $3 \%$ dosage and higher is almost 0.0, and the molecules are stuck on the surface. To demonstrate more clearly how hydrogenated and oxygenated change the diffusion properties for SARA molecules, both sets of results have been shown together in Figure 7c, with a dashed line representing hydrogenated CNTs and the solid line representing oxygenated CNTs. It can be seen that the functionalization with oxygen significantly reduces the diffusion of SARA molecules on the surface. At the same time, a higher dosage of hydrogenation is required for the same diffusion coefficient (Figure 7c). Previous studies show that the diffusion coefficient of SARA molecules and asphalt binder on the aggregates are in the range of $0.8-12 \times 10^{-10} \mathrm{~m}^{2} / \mathrm{s}[12,29,30]$, which is in the same order as our results for a lower dosage of functionalization. Similar to previous studies, we observed that saturate molecule has the highest diffusion coefficient among SARA molecules [30,31]. The obtained adhesion values range from previously reported values for the pure CNT-SARA interface [10].

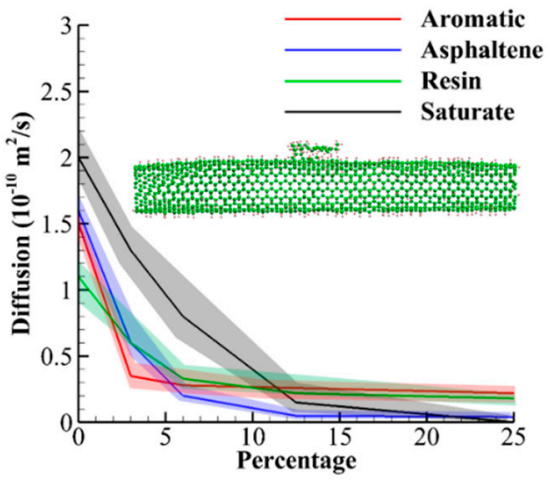

(a)

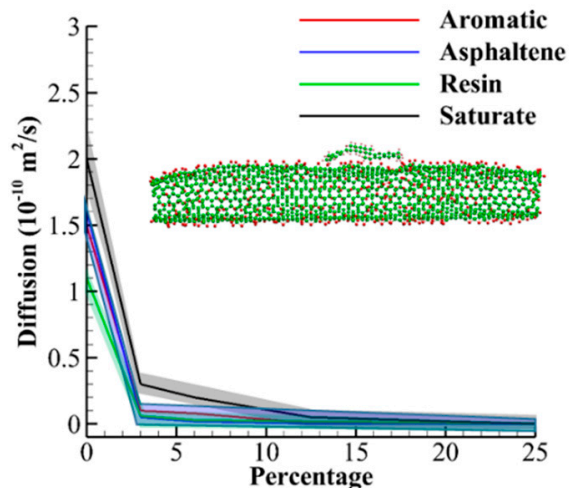

(b)

Figure 7. Cont. 


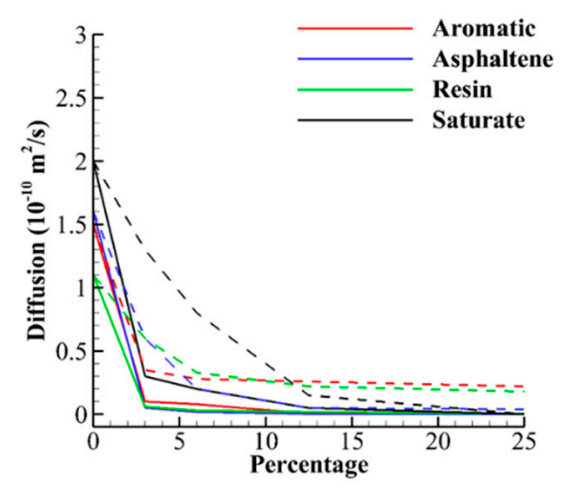

(c)

Figure 7. Diffusion of SARA and different dosages of hydrogenated and oxygenated CNTs. (a) SARAhydrogenated CNTs, (b) SARA-oxygenated CNTs, and (c) comparison of SARA-hydrogenated (dashed lines) and SARA-oxygenated CNTs (solid lines).

\section{Conclusions}

This work evaluated hydrogenated and oxygenated CNTs on the adhesion and diffusion of SARA-CNTs through reactive molecular dynamics simulations. First, we used reactive MD simulation using REAXFF to construct $3 \%, 6 \%, 12 \%$, and $25 \%$ hydrogenated and oxygenated CNTs by randomly pouring hydrogens and oxygens on the surface of CNTs and letting them form bonds with carbon atoms of CNTs. After the construction of hydrogenated and oxygenated CNTs, the SARA molecules are placed on the vicinity of the surface of CNTs and equilibrated for 1ns. Then, the adhesion energy and diffusion coefficient of the CNT-SARA molecules are calculated using SMD and Einstein formula, respectively. The results are then compared to understand the diffusivity and adhesion of SARA molecules on hydrogenated and oxygenated CNTs. The main outcomes of this study are as follow:

- The hydrogenation has little effect on the adhesion energy, but oxygenation can increase adhesion energy significantly.

- Saturate molecule shows the highest increase in adhesion strength from $30 \mathrm{kcal} / \mathrm{mol}$ at $0 \%$ to $60 \mathrm{kcal} / \mathrm{mol}$ for $25 \%$ dosage oxygenation.

- For both hydrogenated and oxygenated CNTs at different dosage, asphaltene, resin, aromatic, and saturate molecules have the highest to lowest values, respectively.

- The diffusion coefficient of SARA molecules on both hydrogenated and oxygenated surfaces drops significantly, with lower values and more sudden drops for the latter.

- The hierarchy of diffusion coefficient values is almost the opposite of adhesion values due to larger interatomic forces; the molecules have less mobility. However, the shape of the molecule may influence the result, and more flexible molecules have more mobilities.

- The values of diffusion coefficient for asphaltene, resin, and aromatic on oxygenated CNTs with $3 \%$ dosage and higher is almost 0.0 , and the molecules are stuck on the surface.

- In experiments, the surface of graphene and CNT oxides are coated with a combination of oxygen and hydroxyl $(\mathrm{OH})$. Therefore this study should be seen as extreme cases where only oxygen/hydrogen exists on the surface. However, this separation reveals that oxygen has the most significant effect on adhesion and diffusion.

Author Contributions: Conceptualization, M.S.; methodology, M.S., H.S.E. and M.R.P.; software, M.S.; validation, M.S., H.S.E. and M.R.P.; formal analysis, M.S.; investigation, M.S.; resources, M.R.P.; data curation, M.S.; writing—original draft preparation, M.S.; writing—review and editing, M.S., H.S.E. and M.P; visualization, H.S.E.; supervision, M.R.P.; project administration, M.S.; funding acquisition, M.R.P. All authors have read and agreed to the published version of the manuscript.

Funding: This research received no external funding. 


\section{Data Availability Statement: Not applicable.}

Conflicts of Interest: The authors declare no conflict of interest.

\section{References}

1. Hiremath, N.; Mays, J.; Bhat, G. Recent Developments in Carbon Fibers and Carbon Nanotube-Based Fibers: A Review. Polym. Rev. 2017, 57, 339-368. [CrossRef]

2. Karousis, N.; Tagmatarchis, N.; Tasis, D. Current progress on the chemical modification of carbon nanotubes. Chem. Rev. 2010, 110, 5366-5397. [CrossRef]

3. Merum, S.; Veluru, J.B.; Seeram, R. Functionalized carbon nanotubes in bio-world: Applications, limitations and future directions. Mater. Sci. Eng. B Solid-State Mater. Adv. Technol. 2017, 223, 43-63. [CrossRef]

4. Shi, M.X.; Li, Q.M.; Huang, Y. Internal resonance of vibrational modes in single-walled carbon nanotubes. Proc. R. Soc. A Math. Phys. Eng. Sci. 2009, 465, 3069-3082. [CrossRef]

5. Strozzi, M.; Pellicano, F. Nonlinear Resonance Interaction between Conjugate Circumferential Flexural Modes in Single-Walled Carbon Nanotubes. Shock Vib. 2019, 2019, 3241698. [CrossRef]

6. Strozzi, M.; Smirnov, V.V.; Manevitch, L.I.; Pellicano, F. Nonlinear normal modes, resonances and energy exchange in single-walled carbon nanotubes. Int. J. Non. Linear. Mech. 2020, 120, 103398. [CrossRef]

7. Duch, J.; Mazur, M.; Golda-Cepa, M.; Podobiński, J.; Piskorz, W.; Kotarba, A. Insight into modification of electrodonor properties of multiwalled carbon nanotubes via oxygen plasma: Surface functionalization versus amorphization. Carbon N. Y. 2018, 137, 425-432. [CrossRef]

8. Ding, Y.; Alias, H.; Wen, D.; Williams, R.A. Heat transfer of aqueous suspensions of carbon nanotubes (CNT nanofluids). Int. J. Heat Mass Transf. 2006, 49, 240-250. [CrossRef]

9. Muthumariappan, A.; Govindasamy, M.; Chen, S.M.; Sakthivel, K.; Mani, V.; Chen, T.W.; Selvaraj, S. Determination of NonSteroidal Anti-Inflammatory Drug (NSAID) azathioprine in human blood serum and tablet samples using Multi-Walled Carbon Nanotubes (MWCNTs) decorated manganese oxide microcubes composite film modified electrode. Int. J. Electrochem. Sci. 2017, 12, 7446-7456. [CrossRef]

10. Sakthivel, K.; Govindasamy, M.; Chen, S.M.; Muthumariappan, A.; Mani, V.; Chen, T.W.; Selvaraj, S. MWCNTs/MoS2 decorated cobalt oxide polyhedrons composite film modified electrode for electrochemical determination of dopamine in rat brain and human blood serum samples. Int. J. Electrochem. Sci. 2017, 12, 7435-7445. [CrossRef]

11. Mirabimoghadam, M.H.; Goli, A.; Molayem, M.; Ameri, M. Experimental study on the effect of nanosized carbon particles on fatigue resistance of asphalt binders. Pet. Sci. Technol. 2016, 34, 971-975. [CrossRef]

12. Ashish, P.K.; Singh, D. Development of empirical model for predicting G*/Sin $\delta$ and viscosity value for nanoclay and Carbon Nano Tube modified asphalt binder. Constr. Build. Mater. 2018, 165, 363-371. [CrossRef]

13. Moreno-Navarro, F.; Sol-Sánchez, M.; Gámiz, F.; Rubio-Gámez, M.C. Mechanical and thermal properties of graphene modified asphalt binders. Constr. Build. Mater. 2018, 180, 265-274. [CrossRef]

14. Pouranian, M.R.; Shishehbor, M. Sustainability assessment of green asphalt mixtures: A review. Environments 2019, 6, 73. [CrossRef]

15. Wang, R.; Qi, Z.; Li, R.; Yue, J. Investigation of the effect of aging on the thermodynamic parameters and the intrinsic healing capability of graphene oxide modified asphalt binders. Constr. Build. Mater. 2020, 230, 116984. [CrossRef]

16. Ramezani, M.G.; Rickgauer, J. Understanding the adhesion properties of carbon nanotube, asphalt binder, and mineral aggregates at the nanoscale: A molecular dynamics study. Pet. Sci. Technol. 2020, 38, 28-35. [CrossRef]

17. Xu, Z.; Wang, Y.; Cao, J.; Chai, J.; Cao, C.; Si, Z.; Li, Y. Adhesion between asphalt molecules and acid aggregates under extreme temperature: A ReaxFF reactive molecular dynamics study. Constr. Build. Mater. 2021, 285, 122882. [CrossRef]

18. Nie, F.; Jian, W.; Lau, D. An atomistic study on the thermomechanical properties of graphene and functionalized graphene sheets modified asphalt. Carbon N. Y. 2021, 182, 615-627. [CrossRef]

19. Amin, I.; El-Badawy, S.M.; Breakah, T.; Ibrahim, M.H.Z. Effect of Functionalization and Mixing Process on the Rheological Properties of Asphalt Modified with Carbon Nanotubes. Am. J. Civ. Eng. Archit. 2016, 4, 90-97.

20. Huang, M.; Zhang, H.; Gao, Y.; Wang, L. Study of diffusion characteristics of asphalt-aggregate interface with molecular dynamics simulation. Int. J. Pavement Eng. 2021, 22, 319-330. [CrossRef]

21. Yao, H.; Dai, Q.; You, Z. Investigation of the asphalt-aggregate interaction using molecular dynamics. Pet. Sci. Technol. 2017, 35, 586-593. [CrossRef]

22. Ellul, M.D.; Gent, A.N. Role of Molecular Diffusion in the Adhesion of Elastomers. J. Polym. Sci. Part A-2 Polym. Phys. 1984, 22, 1953-1968. [CrossRef]

23. Xu, W.; Qiu, X.; Xiao, S.; Hu, G.; Wang, F.; Yuan, J. Molecular dynamic investigations on the adhesion behaviors of asphalt mastic-aggregate interface. Materials 2020, 13, 5061. [CrossRef] [PubMed]

24. Cui, W.; Huang, W.; Xiao, Z.; Xie, J.; Hu, B.; Cai, X.; Wu, K. The Effect of Moisture on the Adhesion Energy and Nanostructure of Asphalt-Aggregate Interface System Using Molecular Dynamics Simulation. Molecules 2020, 25, 4165. [CrossRef]

25. Luo, D.; Guo, M.; Tan, Y. Molecular simulation of minerals-asphalt interfacial interaction. Minerals 2018, 8, 176. [CrossRef]

26. Qu, X.; Wang, D.; Wang, L.; Huang, Y.; Hou, Y.; Oeser, M. The State-of-the-Art Review on Molecular Dynamics Simulation of Asphalt Binder. Adv. Civ. Eng. 2018, 2018, 4546191. [CrossRef] 
27. Shishehbor, M.; Pouranian, M.R.; Imaninasab, R. Evaluating the adhesion properties of crude oil fractions on mineral aggregates at different temperatures through reactive molecular dynamics. Pet. Sci. Technol. 2018, 36, 2084-2090. [CrossRef]

28. Liu, J.; Yu, B.; Wang, S.; Li, L.; Zhang, J. Use of tricalcium silicate to evaluate asphalt absorption on steel slag: Atomic simulation and micro-scale characterization. Meas. J. Int. Meas. Confed. 2021, 177, 109224. [CrossRef]

29. Ma, X.; Wu, J.; Liu, Q.; Ren, W.; Oeser, M. Molecular dynamics simulation of the bitumen-aggregate system and the effect of simulation details. Constr. Build. Mater. 2021, 285, 122886. [CrossRef]

30. Shishehbor, M.; Pouranian, M.R.; Ramezani, M.G. Molecular investigations on the interactions of graphene, crude oil fractions and mineral aggregates at low, medium and high temperatures. Pet. Sci. Technol. 2019, 37, 804-811. [CrossRef]

31. Zhou, X.; Zhang, X.; Xu, S.; Wu, S.; Liu, Q.; Fan, Z. Evaluation of thermo-mechanical properties of graphene/carbon-nanotubes modified asphalt with molecular simulation. Mol. Simul. 2017, 43, 312-319. [CrossRef]

32. Ismael, M.Q.; Fattah, M.Y.; Jasim, A.F. Improving the rutting resistance of asphalt pavement modified with the carbon nanotubes additive. Ain Shams Eng. J. 2021. [CrossRef]

33. Pei, S.; Cheng, H.M. The reduction of graphene oxide. Carbon N. Y. 2012, 50, 3210-3228. [CrossRef]

34. Li, D.D.; Greenfield, M.L. Chemical compositions of improved model asphalt systems for molecular simulations. Fuel 2014, 115, 347-356. [CrossRef]

35. Plimpton, S. Fast parallel algorithms for short-range molecular dynamics. J. Comput. Phys. 1995, 117, 1-19. [CrossRef]

36. Van Duin, A.C.T.; Dasgupta, S.; Lorant, F.; Goddard, W.A. ReaxFF: A reactive force field for hydrocarbons. J. Phys. Chem. A 2001, 105, 9396-9409. [CrossRef]

37. Humphrey, W.; Dalke, A.; Schulten, K. VMD: Visual molecular dynamics. J. Mol. Graph. 1996, 14, 33-38. [CrossRef]

38. Park, S.; Khalili-Araghi, F.; Tajkhorshid, E.; Schulten, K. Free energy calculation from steered molecular dynamics simulations using Jarzynski's equality. J. Chem. Phys. 2003, 119, 3559-3566. [CrossRef] 